\title{
Virtual Laboratory in Mechanisms Design
}

\author{
Oleg Vinogradov \\ Department of Mechanical and Manifacturing Engineering \\ University of Calgary \\ ovinogra@ucalgary.ca
}

\begin{abstract}
A concept of virtual laboratory as a tool to teach kinematics and dynamics of mechanisms is presented. A virtual laboratory has many advantages vs physical laboratory: it is not limited to a specific variety of mechanisms, it allows to "measure" the trajectory, velocity and acceleration of any point, it allows to "measure" internal forces in the joints, and, what is most important, it allows changing the links dimensions and observe the consequences in terms of the mechanism's output function. An important component of the virtual lab is the motion animation, which allows student to develop a visual perception and intuition.
\end{abstract}

\section{Introduction}

Kinematics is the study of motion in mechanisms: position, displacement, rotation, velocity, and acceleration. Traditionally, the only way to study such motion was to design and manufacture a physical prototype and run it in the lab. In this setting, the displacements, velocities, accelerations, and forces had to be measured. This physical simulation is inflexible in terms of mechanisms parameters, and, more importantly, due to costs and space limitations, the variety of mechanisms available for the study is limited. In addition, the study of mechanisms in a physical lab is usually done in groups, which limits a student's personal experience due to time constraints.
With the advent of computers the utilization of software for kinematical and dynamic analysis of mechanisms became a possibility and opened new avenue for students learning. The first programs were written in FORTRAN and were made available to students [1]. Later symbolic languages such as MATLAB [2] and MATHEMATICA [3] were utilized for the mechanisms analysis. The advantage of using symbolic languages was that it allowed students to write their own programs in a relatively short time and thus provided more flexibility in learning. An example of mechanisms studied (such as Dump Truck, Double-Toggle Punch, Variable-Drive) can be seen at http://www.enme.ucalgary.ca/courses/enme 473/virtual_lab.zip.

In the following a typical student project in mechanism design is presented. Most mechanisms for the projects are adopted from [4].

\section{Typical Design Projects}

A typical project comprises three components: position analysis, velocity and acceleration analysis, and force analysis. The important characteristic of a project assigned to students is that it iss formulated in terms of functional requirements for the 
mechanism without giving any other velocities, forces, etc. In this approach the project becomes open-ended and the students have to achieve the required functional performance by trial-and-error method. The software they develop allows them to accomplish this in a short time. In their reports students, among other requirements, must do the following for one cycle of the input link motion:

1. Plot positions, velocities and accelerations of selected points;

2. Animate the motion;

3. Assume the resistance (force) applied to the output link and find the driving torque/force required;

4. Plot the needed driving torque/force and one or two plots of internal forces in selected joints of their choice.

\section{An Adjustable Slide Drive Mechanism}

An example of such a mechanism is shown in Fig.1. It consists of a crankslider with an adjustable frame, which can be lengthened and shortened, in the vertical direction. Input is provided by the rotation of the input crank, and specifications concerning dimensions, output is measured as the lateral motion of the intersection of the slider and a horizontal line, upon which another slider is mounted. Adjusting the frame alters the output motion, even as the input motion remains constant.

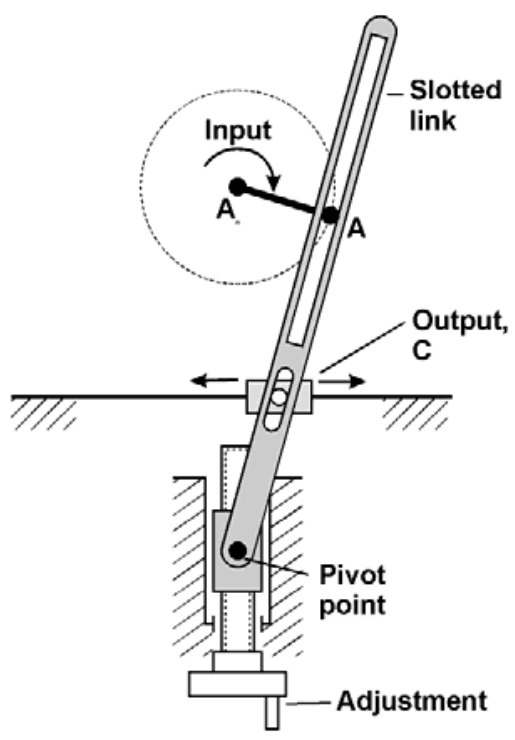

Fig. 1. Adjustable Slide Drive Mechanism

In Fig.2 three snapshots of the mechanism at different positions during one cycle of animation are shown. 

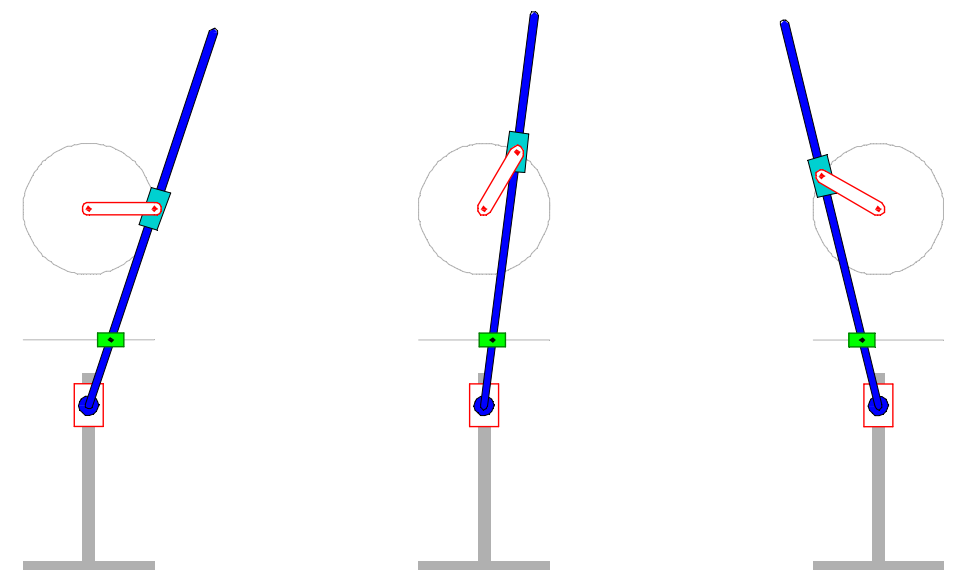

Fig.2. Three snapshots of the Adjustable Slide Drive Mechanism in animation

Variable Drive Mechanism

Another example of a complex mechanism, comprising two four-bar linkages functionally in series, is shown in Fig.3. The first mechanism has its input link connected to a rotating driveshaft, and its output link connected to a moveable pivot point. The second mechanism has as its input the output link from the first mechanism, and its output link is attached to the output shaft with a uni-directional ratchet. By varying the location of the moveable pivot, the motion of the output link can be changed. Typical application of this mechanism is in cars, where it allows an engine to operate at a constant rotational speed, representing the maximum fuel efficiency and/or power output, and yet permits control over the rotational speed of the output shaft.

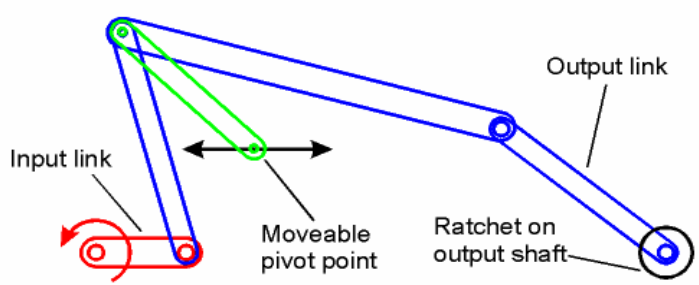

Fig.3. Variable Drive Mechanism

In Fig.4 three snapshots of the Variable Drive Mechanism during one cycle of input link rotation are shown. 


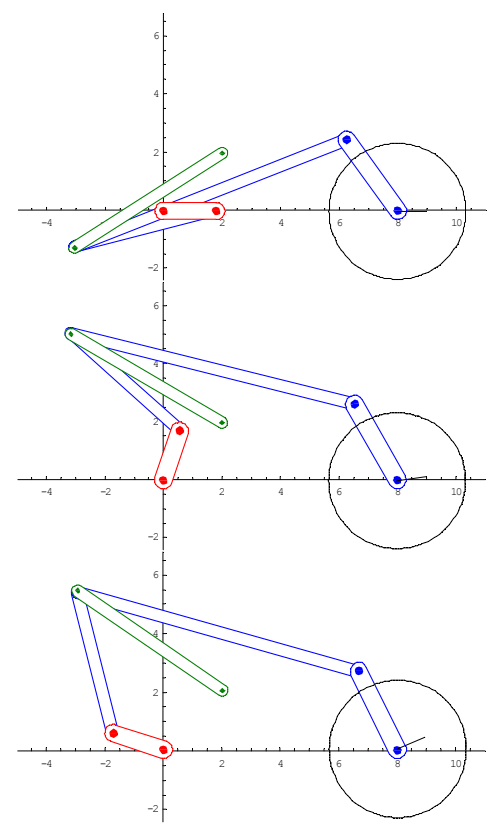

Fig.4. Three snapshot of the Variable Drive Mechanism during one cycle of input link rotation

The basic report requirements are the same as for the Adjustable Slide Drive Mechanism above. In this case, however, the students are supposed to do the analysis of the output velocity for two positions of the movable pivot point.

Cam and Valve mechanism

Another example of a mechanism, involving cam as a driving element, is shown in Fig.5. This mechanism is used in internal combustion engines. The rotation of the cam is transformed through the follower-arm links into a displacement of the valve. As the valve moves down, fuel is allowed to enter the combustion chamber, and then the valve returns to the closed position during combustion.

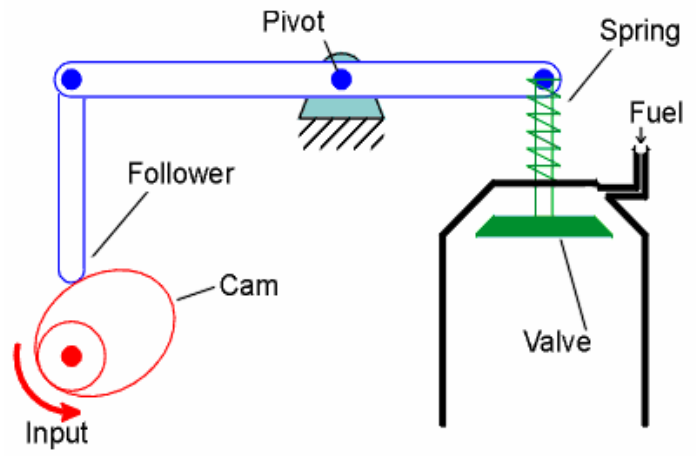

Fig.5 Cam and Valve mechanism
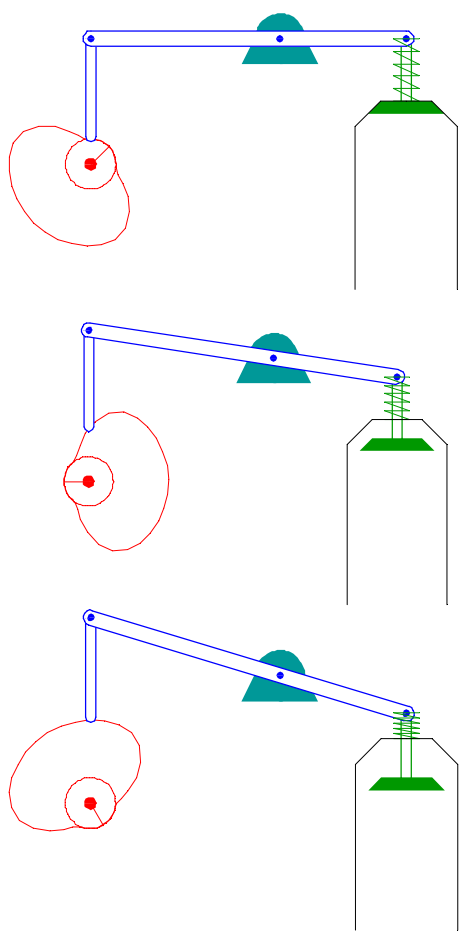

Fig. 6 Three snapshots of animation of the Cam and Valve mechanism 


\section{Conclusion}

In summary, a virtual lab is not only an alternative to a physical set up but in fact extends the possibilities and experiences for students in designing mechanisms. It llows mechanism animation, which is a powerful tool in learning since it provides a visual perception of motion. Students can also immediately see the effect of design decisions on the mechanism motion and performance: point trajectories, velocities, accelerations, and external and internal forces acting on links and joints.

\section{References}

[1] P.E. Nikravesh, Computer-Aided Analysis of Mechanical Systems, Prentice Hall, 1988

[2] K.J. Waldron, G.L. Kinzel, Kinematics, Dynamics and Design of Machinery, Wiley, 1999

[3] O. Vinogradov, Fundamentals of Kinematics and Dynamics of Machines and Mechanisms, CRC Press, 2000

[4] N.P. Chironis, Mechanisms \& Mechanical Devices Sourcebook, McGrawHill, 1991 